\title{
Efficient Sensings of Temperature, Refractive Index, and Distance Measurement using the Cubic- Nonlinear Optoelectronic Oscillators
}

\section{Kevie Marlone Tchuissa Koualong}

University of Dschang Faculty of Sciences: Universite de Dschang Faculte des Sciences

Jimmi Herve Talla Mbe ( $\sim$ jhtallam@yahoo.fr)

Universite de Dschang Faculte des Sciences https://orcid.org/0000-0002-4928-6578

Thomas Tamo Tatietse

University of Yaounde I: Universite de Yaounde I

\section{Research Article}

Keywords: Optoelectronic oscillator (OEO), Oscillation frequency, Temperature sensing, Refractive index sensing, Distance measurement

Posted Date: December 20th, 2021

DOI: https://doi.org/10.21203/rs.3.rs-1162598/v1

License: (c) (i) This work is licensed under a Creative Commons Attribution 4.0 International License. Read Full License

Version of Record: A version of this preprint was published at Optical and Quantum Electronics on April 19th, 2022. See the published version at https://doi.org/10.1007/s11082-022-03702-y. 


\title{
Efficient sensings of temperature, refractive index, and distance measurement using the cubic-nonlinear optoelectronic oscillators
}

\author{
Kevie Marlone Tchuissa Koualong • \\ Jimmi Hervé Talla Mbé . Thomas Tamo \\ Tatietse
}

Received: date / Accepted: date

\begin{abstract}
In this work, we use the cubic-nonlinear optoelectronic oscillator (CNOEO) in three different configurations to successfully perform the sensings of temperature, refractive index, and distance measurement with high precision. From our results, the use of the CNOEO through the variation of the values of the cubic-nonlinear parameter increases the sensitivity of the different sensings and the measurement carried out compared to the results obtained with the standard OEO which is a particular CNOEO featuring a cubic-nonlinear parameter equal to zero.
\end{abstract}

Keywords Optoelectronic oscillator (OEO) • Oscillation frequency • Temperature sensing · Refractive index sensing · Distance measurement

\section{Introduction}

Optoelectronic oscillators (OEOs) have inspired numerous applications and technological aims such as ultra-stable microwave generation, neuromorphic computing, signal processing, photonic integration circuits, random numbers generation, and chaos communications (see refs. [1-4] and references therein

Kevie Marlone Tchuissa Koualong

Research unit of Condensed Matter, Electronics and Signal Processing, Department of Physics, University of Dschang, P.O. Box 67, Dschang, Cameroon

Jimmi Hervé Talla Mbé

Research unit of Condensed Matter, Electronics and Signal Processing, Department of Physics, University of Dschang, P.O. Box 67, Dschang, Cameroon

Tel.: +237-675482406

E-mail: jhtallam@yahoo.fr

Thomas Tamo Tatietse

Department of Civil Engineering, National Advanced School of Engineering,

University of Yaounde I, P.O. Box 510, Cameroon 
for a comprehensive review). Recently, the scope of technology and applications has widened to incorporate sensing, measurement, and detection [5-7] owing to the fact that typically, an OEO is a high-Q device capable of producing microwave signals with ultralow phase noise and high frequency [8]. Indeed, with OEOs it is possible to carry out the measurement or sensing of length, refractive index, temperature, angular velocity, load, strain, lowpower RF signal, magnetic field, amongst others (see Refs. [8-16] and references therein). For this purpose, several architectures were proposed for the efficiency of the method. For instance, the authors of [16] achieved a straininsensitive temperature sensor with a sensitivity of $1.00745 \mathrm{MHz} /{ }^{\circ} \mathrm{C}$ based on an optoelectronic oscillator incorporating both the phase and the intensity optical modulators. Besides, Zhang et al. achieved a sensitivity of the angular velocity of $51.8 \mathrm{kHz} /(\mathrm{rad} / \mathrm{s})$ using an OEO incorporating a Sagnac interferometer [8].

The fundamental principle of using OEO for optical metrology is to code the quantity to evaluate, such as temperature, strain, transverse load, refractive index, and more, to the frequency shift of oscillating signal of OEOs. Two mechanisms are possible [16]: The first one consists of using the band-pass of the microwave photonic filter both as an oscillation frequency selection element as well as a sensing/measurement element. Even if it gives high sensitivity for optical metrology, it is not easy to implement experimentally. The second one known as the time-delay mechanism is quite easy since it relies on the direct change in the oscillator's loop when affected by the quantity to sense, detect, or measure.

Thus, this latter technique is the most developed one [2]. Following this, an OEO with optimal frequency could play an important role in sensing and measurement processes. Recently, an OEO displaying dynamics predominated by high-frequency limit-cycle oscillations was proposed [17]. Such dynamics were made possible by substituting the standard band-pass filter in the electrical path of the standard OEO with a cubic-nonlinear band-pass filter. The oscillator is known as the cubic-nonlinear optoelectronic oscillator (CNOEO) and has not yet been used for sensing and measurement.

In this paper, we theoretically aim to increase the sensitivity of the sensor/measurerbased OEOs using the CNOEO. The distance measurement and two main sensings are investigated, namely, the temperature and the refractive index. The paper is organized as follows: In Sect. 2, the cubic-nonlinear optoelectronic oscillator (CNOEO) is presented. Applications to temperature, refractive index sensings, and the distance measurement are discussed in Sects. 3, 4, and 5 , respectively. Finally, a conclusion is given in Sect. 6 .

\section{The cubic-nonlinear optoelectronic oscillator (CNOEO)}

The CNOEO used for this work is displayed in Fig. 1(a) [17]. The CNOEO is made up of a laser diode, a polarization controller (PC), a Mach-Zehnder 


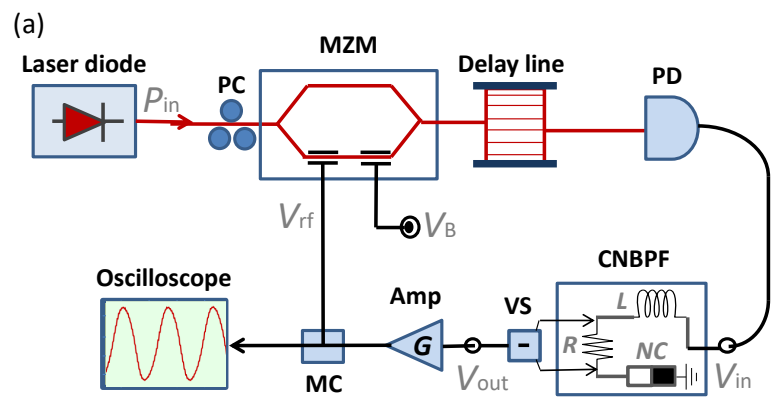

(b)

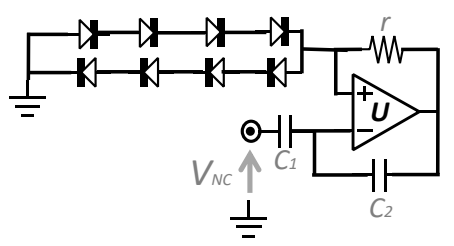

Fig. 1 (a) Circuit design of the cubic-nonlinear optoelectronic oscillator (CNOEO) [17]. PC: Polarization Controller; MZM: Mach-Zehnder Modulator; $V_{B}$ is the offset phase control voltage; PD: Photodiode; CNBPF: Cubic-Nonlinear Band-Pass Filter; VS: Voltage Subtractor; Amp: amplifier; MC: Microwave Coupler. $L$ and $R$ are the coil and the resistor, respectively. (b) The structure of the nonlinear capacitor (NC). $C_{1,2}$ are capacitors.

modulator (MZM), an optical delay line, a photodiode (PD), a cubic-nonlinear band-pass filter (CNBPF), a voltage subtractor (VS), an amplifier (Amp), a microwave coupler (MC), and an oscilloscope to visualize the oscillating signal. The $\mathrm{CNBPF}$ is made of an inductor, a resistance, and a nonlinear capacitor (NC). The structure of the nonlinear capacitor is shown in Fig. 1b: an operational amplifier (U), two capacitors $C_{1,2}$, one resistor $r$, and eight junction diodes can be identified. The functioning of the CNOEO will be specifically given for each application treated in this paper. Detailed explanations of the main CNOEO can be found in Refs. [17,18]. Nevertheless, it is important to notice that the dynamics of the CNOEO is governed by the following integrodelay differential equation $[17,18]$ :

$$
x(t)+\mu \frac{d x(t)}{d t}+\frac{1}{\theta} \int_{0}^{t} x(t) d t+u\left(\int_{0}^{t} x(t) d t\right)^{3}=\beta \cos ^{2}[x(t-\tau)+\phi],
$$


where the parameters are expressed in terms of the components of the system of Fig. 1 as follows:

$$
\left\{\begin{array}{l}
\mu=\frac{L}{R} \\
u=\frac{n_{o} V_{o}}{6}\left(\frac{1}{2 i_{o} R r C_{2}}\right)^{3}\left(\frac{2 V_{\pi r f}}{\pi G}\right)^{2}, \\
\beta=\frac{\pi G P S}{2 V_{\pi r f}} \\
\frac{1}{\theta}=\left[\frac{1}{C_{1}}-\frac{n_{o} V_{o}}{2 i_{o} r C_{2}}\right] \frac{1}{R} \\
x(t-\tau)=\frac{\pi V_{r f}(t-\tau)}{2 V_{\pi r f}} \\
\phi=\frac{\pi V_{B}}{2 V_{\pi d c}},
\end{array}\right.
$$

In the set of Eq. (2), $\mu$ is the high cut-off time, $\theta$ the low cut-off time, $\tau$ the time-delay, $x$ the dimensionless dynamical variable of the system, $n_{o}=$ 4 the number of junction diodes in series and whose characteristics are the thermal voltage $V_{o}=25 \mathrm{mV}$ and inverse saturation current $i_{o}=5 \mu \mathrm{A}$. $u$ is the cubic-nonlinear coefficient. $V_{\pi r f}, V_{\pi d c}$, and $V_{B}$ are the radio-frequency (rf), the direct-current $(\mathrm{dc})$ half-wave voltages, and the bias voltage of the MachZehnder modulator, respectively. The gain of the amplifier is $G$, the sensitivity of the photodiode $S$, and $P$ is the power of the laser diode. The values of these parameters are taken compatible with the experiment $[17,18]: V_{\pi r f}=3.8 \mathrm{~V}$, $V_{\pi d c}=5 \mathrm{~V}, R=2.5 \mathrm{k} \Omega, r=300 \Omega, L=0.1 \mathrm{mH}, C_{1}=270 \mathrm{pF}, C_{2}=9.15 \mathrm{nF}$, $S=4.75 \mathrm{~V} / \mathrm{mW}$.

When using OEOs for sensings, detections, and measurements, the oscillation limit-cycle frequency plays a fundamental role since the principles are based on the variation of this frequency recorded on an oscilloscope [2]. It was demonstrated that due to the cubic-nonlinear term $u\left(\int_{0}^{t} x(t) d t\right)^{3}$ in Eq.1, CNOEO features limit-cycle oscillations of higher frequency $f_{\text {osc }}$ compared to the standard OEO, yielding $[17,18]$ :

$$
f_{\text {osc }}=f_{s d}\left(1+\frac{3 \rho y_{s t}^{2}}{\alpha}\right)^{\frac{1}{2}},
$$

where $f_{s d}$ is the oscillating frequency of the standard OEO, $\rho=u \mu^{3}$ the cubic-nonlinear parameter, and $\alpha=\frac{\mu}{\theta}$ the cut-off times ratio. Considering the following variable $y=\int_{0}^{t} x d s, y_{s t}$ denotes the corresponding fixed point of our system. It is important to note that the CNOEO is identified by $\rho \neq 0$, whilst for the standard OEO, $\rho=0$. Experimentally, $\rho$ can be turned through the gain $\mathrm{G}$ of the amplifier (see Eq. (2)). The next sections (Sect. 3, 4, and 5) deal with some sensings and one measurement using the CNOEO.

\section{Temperature sensing using CNOEO}

\subsection{Circuit and principle}

The setup for temperature sensing is given in Fig. 2. As in Refs. [5, 19], the single-mode fiber (SMF) is the temperature sensor. Indeed, the SMF is sub- 


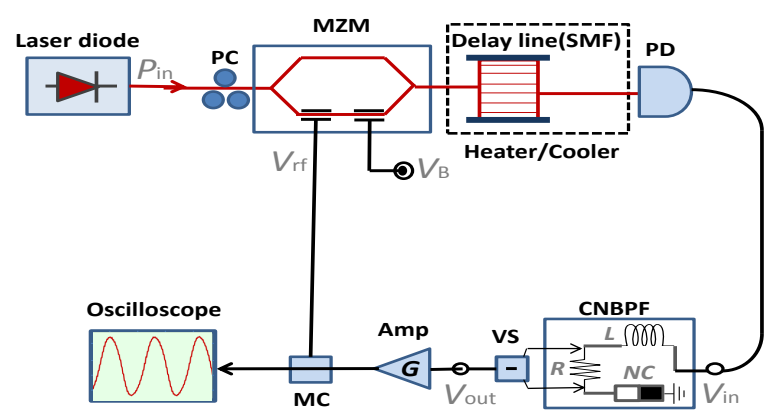

Fig. 2 Setup of the temperature sensing using a CNOEO. SMF: Single-mode fiber.

jected to different temperatures by heating or cooling as shown in Fig. 2. The variation of temperature consequently modifies the total time-delay $\tau$ of the CNOEO which is given by:

$$
\tau=\tau_{o p}+\tau_{1},
$$

with $\tau_{o p}$ being the proper time-delay of the SMF which corresponds to the time needed by light to travel through the SMF and $\tau_{1}$ is the time-delay of the remaining path of the CNOEO's loop, including both the electrical and optical paths. Noting that each time-delay $\tau_{o p}$ and $\tau_{1}$ is related to a length $l_{o p}$ and $l_{1}$, through the speed of the signal in the SMF and other paths of the CNOEO loop, respectively. Thus, the frequency is a function of the total length $l=l_{o p}+l_{1}$ and the refractive index $n$, and yields [19]:

$$
f=\frac{c}{2 \pi n l}
$$

Equation (5) gives the frequency of the signal recorded at the oscilloscope. Indeed, the frequency $f$ is a sum of the nominal value $f_{\text {osc }}$ (see Eq. (3)) and the variation $\Delta f$ due to the variation of temperature $(\Delta T)$ sensed by the SMF; that is:

$$
f=f_{\text {osc }}+\Delta f
$$

In OEOs, the length $l$ and refractive index $n$ of the SMF are the two parameters that vary with the temperature. Differentiating Eq. (5) with respect to $n$ and $l$, and assuming that $l_{o p}>>l_{1}$, the variation of the frequency $\Delta f$ due to heating/cooling yields:

$$
\Delta f=-f_{s d} \frac{l_{o p}}{l}\left(\alpha_{1}+\xi\right)\left(1+\frac{3 \rho y_{s t}^{2}}{\alpha}\right)^{\frac{1}{2}} \Delta T
$$


In Eq. (7), $\Delta f$ is the variation of the frequency recorded by the oscilloscope, $\Delta T$ the variation of the temperature, $\alpha_{1}$ the expansion coefficient, and $\xi$ the thermo-optic coefficient of the SMF [19]. $\Delta T$ also characterizes the temperature sensitivity and precision; the more $\Delta T$ is smaller, the more our CNOEO can detect a slight temperature change.

\subsection{Results}

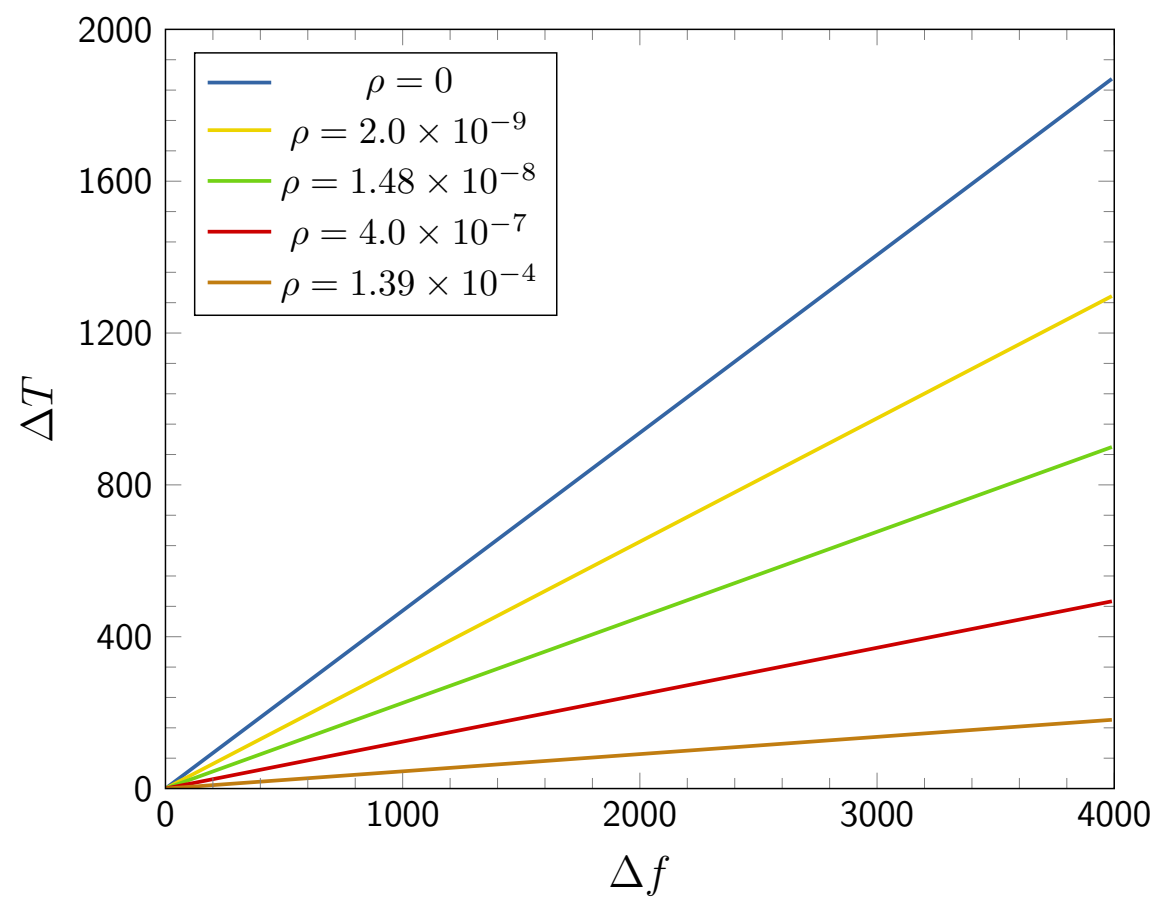

Fig. 3 Temperature variation $\Delta T$ versus frequency variation $\Delta f$. From up to down, $\rho=0$, $\rho=2 \times 10^{-9}, \rho=1.48 \times 10^{-8}, \rho=4 \times 10^{-7}$, and $\rho=1.39 \times 10^{-4}$.

Figure 3 shows the variation of the temperature $\Delta T$ against the variation of the recorded frequency in the oscilloscope $\Delta f$ (see Eq. (7)). Figure 3 is the superposition of 5 plots obtained by varying $\rho$. Each plot of Fig. 3 is a linear variation with a positive slope which decreases as the cubic-nonlinear parameter $\rho$ increases. From up to down, the curve is obtained for $\rho=0$, $\rho=2.0 \times 10^{-9}, \rho=1.48 \times 10^{-8}, \rho=4 \times 10^{-7}$, and $\rho=1.39 \times 10^{-4}$. It can be noticed that for a given variation of the frequency recorded in the oscilloscope, the value of the change in temperature sensed by the SMF decreases with the increase of the cubic-nonlinear parameter $\rho$ (see Fig. 3). Some cases are illustrated in Tab. 1. For instance, for a recorded value of $\Delta f=1050$, the 


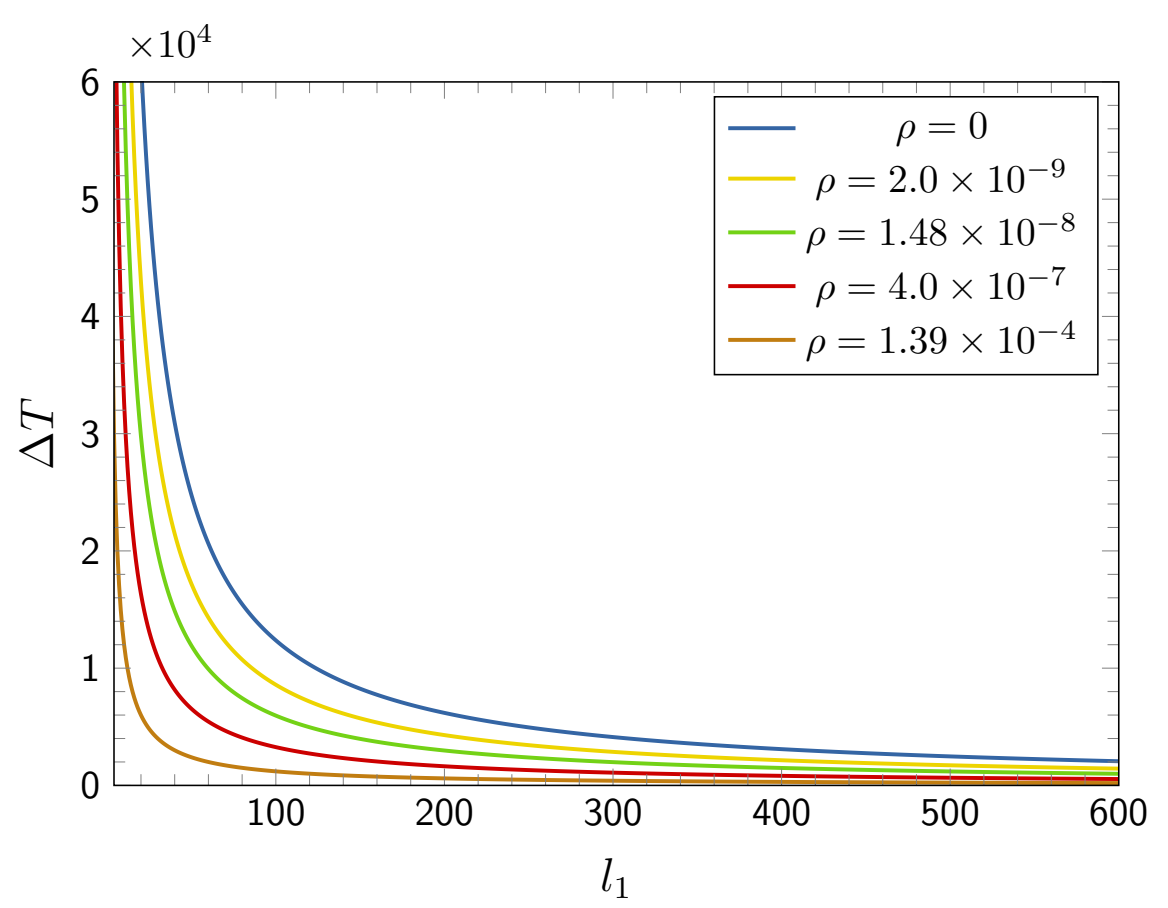

Fig. 4 Temperature variation versus length (plotted from Eq. (7)). From up to down, $\rho=0$, $\rho=2 \times 10^{-9}, \rho=1.48 \times 10^{-8}, \rho=4 \times 10^{-7}$, and $\rho=1.39 \times 10^{-4}$.

standard OEO displays a variation of the temperature of $\Delta T=492$ which is less precise compared to $\Delta T=341.4, \Delta T=236.8, \Delta T=129.8$, and $\Delta T=46.66$ obtained with the CNOEO when the cubic-nonlinear parameter is increasingly monitored to the values $\rho=2.0 \times 10^{-9}, \rho=1.48 \times 10^{-8}$, $\rho=4 \times 10^{-7}$, and $\rho=1.39 \times 10^{-4}$, respectively. Noting that the higher precision (smaller value of $\Delta T$ ) is obtained with the higher value of $\rho$; that is $\Delta T=46.66$ for $\rho=1.39 \times 10^{-4}$. A similar example showing the decrease of $\Delta T$ when $\rho$ grows is obtained for $\Delta f=1500$ (see Tab. 1 ).

Table 1 Corresponding values of $\Delta T$ at fixed $\Delta f$ for different values of $\rho$.

\begin{tabular}{lllll}
\hline Nonlinear parameter $(\rho)$ & $\Delta f$ & $\Delta T$ & $\Delta f$ & $\Delta T$ \\
\hline$\rho=0$ & 1050 & 492 & 1500 & 702.9 \\
\hline$\rho=2.0 \times 10^{-9}$ & 1050 & 341.4 & 1500 & 487.7 \\
$\rho=1.48 \times 10^{-8}$ & 1050 & 236.8 & 1500 & 338.2 \\
$\rho=4.0 \times 10^{-7}$ & 1050 & 129.8 & 1500 & 185.4 \\
$\rho=1.39 \times 10^{-4}$ & 1050 & 46.66 & 1500 & 68.1 \\
\hline
\end{tabular}

Figure 4 displays the variation of the temperature against the length $\left(l_{1}\right)$. It is also a superposition of 5 graphs as that shown in Fig. 3. But, it is an inverse variation (as Eq. (7) demonstrates) which values decrease as the cubic- 
nonlinear parameter $\rho$ is being increased (see Fig. 4). Table 2 shows one numerical illustration. Here, one still observes the same sensitivity of $\Delta T$ as $\rho$

Table 2 Corresponding values of $\Delta T$ at fixed $l_{1}$ for different values of $\rho$.

\begin{tabular}{lll}
\hline Nonlinear parameter $(\rho)$ & $l_{1}$ & $\Delta T$ \\
\hline$\rho=0$ & 100 & $1.237 \times 10^{4}$ \\
\hline$\rho=2.0 \times 10^{-9}$ & 100 & $8.584 \times 10^{3}$ \\
$\rho=1.48 \times 10^{-8}$ & 100 & $5.953 \times 10^{3}$ \\
$\rho=4 \times 10^{-7}$ & 100 & $3.264 \times 10^{3}$ \\
$\rho=1.39 \times 10^{-4}$ & 100 & $1.198 \times 10^{3}$ \\
\hline
\end{tabular}

increases. For example, when $l_{1}$ is equal to $100, \Delta T=1.237 \times 10^{4}$ for the standard OEO $(\rho=0)$, whereas it is significantly reduced to $\Delta T=3.264 \times 10^{3}$ for $\rho=4 \times 10^{-7}$ and $\Delta T=1.198 \times 10^{3}$ for $\rho=1.39 \times 10^{-4}$. Thus, this demonstrates the fact that $\rho$ increases the sensitivity of our CNOEO as a temperature sensor.

\section{Refractive index sensing using a CNOEO}

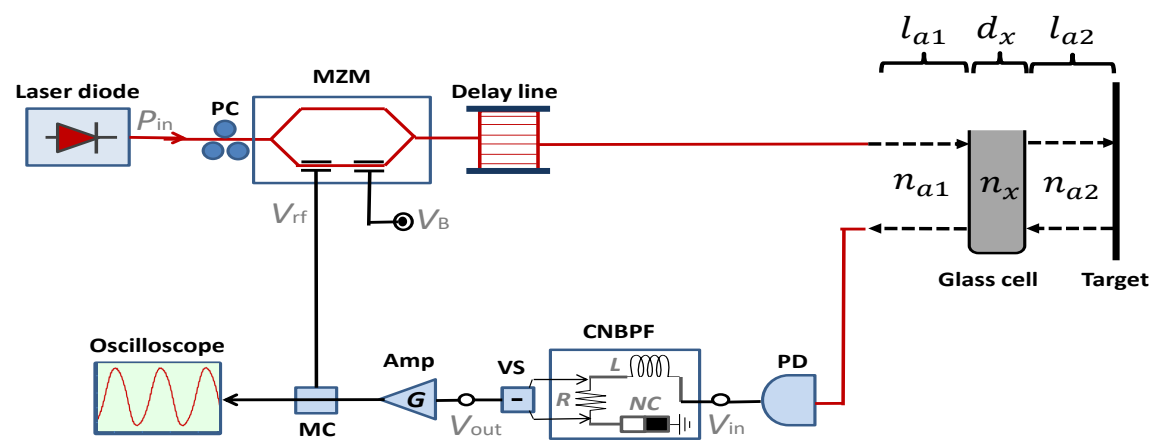

Fig. 5 Setup of the refractive index sensing using a CNOEO. $l_{a 1}$ is the first free space distance of refractive index $n_{a 1}, l_{a 2}$ the second free space distance of refractive index $n_{a 2}$, $d_{x}$ represents the diameter of the glass cell of refractive index $n_{x}$. 
4.1 Circuit and principle

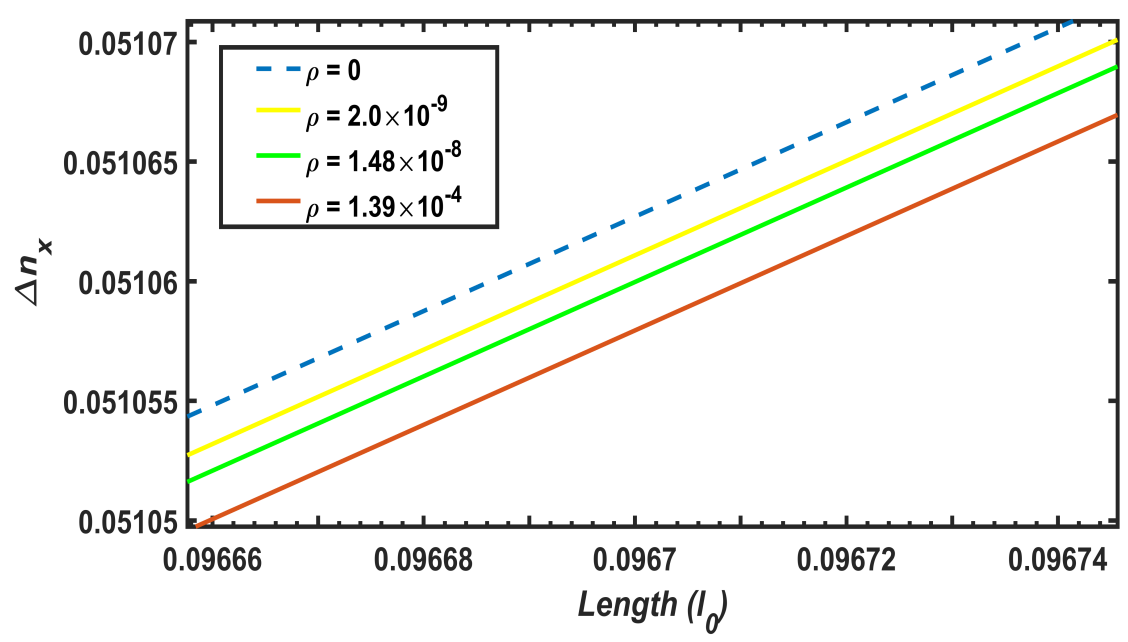

Fig. 6 Variation of the refractive index versus the length. From up to down, $\rho=0, \rho=$ $2 \times 10^{-9}, \rho=1.48 \times 10^{-8}$, and $\rho=1.39 \times 10^{-4}$.

Based on Ref. [20], the proposed setup used for the refractive index sensing is shown in Fig. 5. The liquid whose refractive index $n_{x}$ is to determine is inserted in the glass cell of diameter $d_{x}$. The light from the optical delay line passes through the first free space, then the glass cell, and after, the second free space. It hits the target and is reflected again to the same path. The extra time-delay due to the liquid will cause a variation of the oscillation frequency recorded on the oscilloscope. This oscillation frequency will be used to evaluate the refractive index $n_{x}$. Indeed, the total time-delay $\tau$ of the signal to come to completion is the sum of the electrical time-delay $\left(\tau_{e}\right)$ and the optical timedelay $\left(\tau_{\text {opt }}\right)$ given by [21]:

$$
\tau=\tau_{e}+\tau_{o p t}
$$

Let us note the length of the free space $l_{a}=l_{a 1}+l_{a 2}$ with the refractive index $n_{a}=n_{a 1}=n_{a 2}$, which is the refractive index of air. The mathematical expression of $\tau_{o p t}$ is given below:

$$
\tau_{\text {opt }}=\frac{n_{1} l_{0}}{c}+\frac{2 n_{a} l_{a}}{c}+\frac{2 n_{x} d_{x}}{c},
$$

where $l_{0}$ is the total length of the optical delay line in Fig. $5, n_{1}$ its refractive index, and c the speed of light in the vacuum. In Eq. (9), 2 accounts for the round trip of light in the media of refractive indexes $n_{a 1}, n_{x}$, and $n_{a 2}$ (see Fig. 5). Equation (8) becomes [22]:

$$
\tau=\tau_{e}+\frac{n_{1} l_{0}}{c}+\frac{2 n_{a} l_{a}}{c}+\frac{2 n_{x} d_{x}}{c}
$$


Let us consider $n_{1} L_{1}=n_{1} l_{0}+c \tau_{e}$. It implies that Eq. (10) becomes

$$
\tau c=n_{1} L_{1}+2 n_{a} l_{a}+2 n_{x} d_{x}
$$

By definition, the oscillation frequency $f_{o s c}=\frac{k}{\tau}$, with $k$ an integer standing for the mode number ( $k=1$ for the fundamental mode) [21,22]. Thus,

$$
f_{\text {osc }}=\frac{k c}{n_{1} L_{1}+2 n_{a} l_{a}+2 n_{x} d_{x}}
$$

Differentiating Eq. (12), the variation of frequency is given by:

$$
\Delta f_{o s c}=-\frac{2 f_{o s c} \cdot F S R \cdot d_{x}}{c} \Delta n_{x}
$$

Equation (13) is similar to the one obtained by [22]. Here, FSR is the free spectral range which expression is given by:

$$
F S R=\frac{c}{n_{1} L_{1}+2 n_{a} l_{a}+2 n_{x} d_{x}}=\frac{1}{\tau}
$$

After the substitutions of FSR and Eq. (3) in Eq. (13), one obtains the mathematical expression of the variation of the refractive index given below:

$$
\Delta n_{x}=-\frac{n_{1} l_{0}+\frac{c}{f_{s d}}\left(1+\frac{3 \rho y_{s t}^{2}}{\alpha}\right)^{-\frac{1}{2}}+2 n_{a} l_{a}+2 n_{x} d_{x}}{2 f_{o s c} \cdot d_{x}} \Delta f_{o s c}
$$

Finally, the refractive index $n_{x}$ of the liquid is computed through:

$$
n_{x}=n_{a}+\Delta n_{x}
$$

\subsection{Results}

Figure 6 plots the variation of the refractive index against the optical length $\left(l_{0}\right)$. It can be seen that the variation of the refractive index $\Delta n_{x}$ reduces as one keeps on increasing $\rho$ for a given value of optical length $l_{0}$. This variation is very small since it is in the order of five digits showing then the high sensitivity of our system. That sensitivity is more accurate when the cubicnonlinear parameter $\rho$ becomes more important. From up to down, the curves are obtained for $\rho=0$ (standard OEO), $\rho=2.0 \times 10^{-9}, \rho=1.48 \times 10^{-8}$, and $\rho=1.39 \times 10^{-4}$.

In Fig. 7, we also show the variation of the refractive index $\Delta n_{x}$ against the variation of the frequency $\Delta f_{\text {osc }}$ (see Eq. (15)). Here also, one remarks that with higher values of the cubic-nonlinear parameter $\rho$, higher precision $\Delta n_{x}$ in the values of the refractive index are obtained. Moreover, $\Delta n_{x}$ is reduced to the order of 8 digits. 


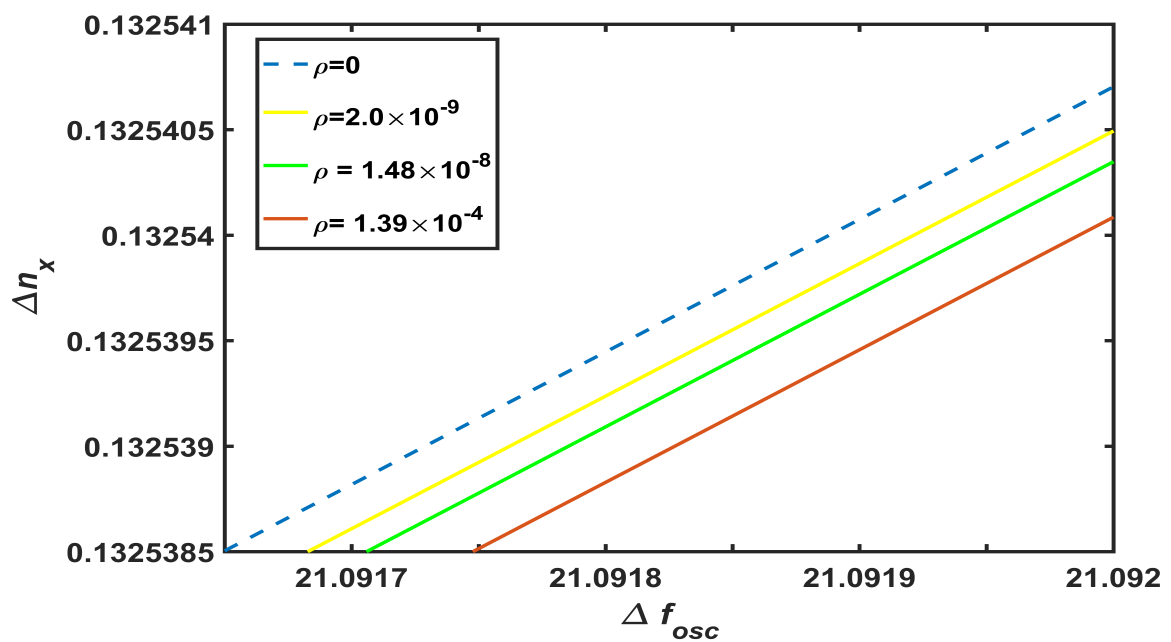

Fig. 7 Variation of the refractive index versus the variation of the frequency. From up to down, $\rho=0, \rho=2 \times 10^{-9}, \rho=1.48 \times 10^{-8}$, and $\rho=1.39 \times 10^{-4}$.

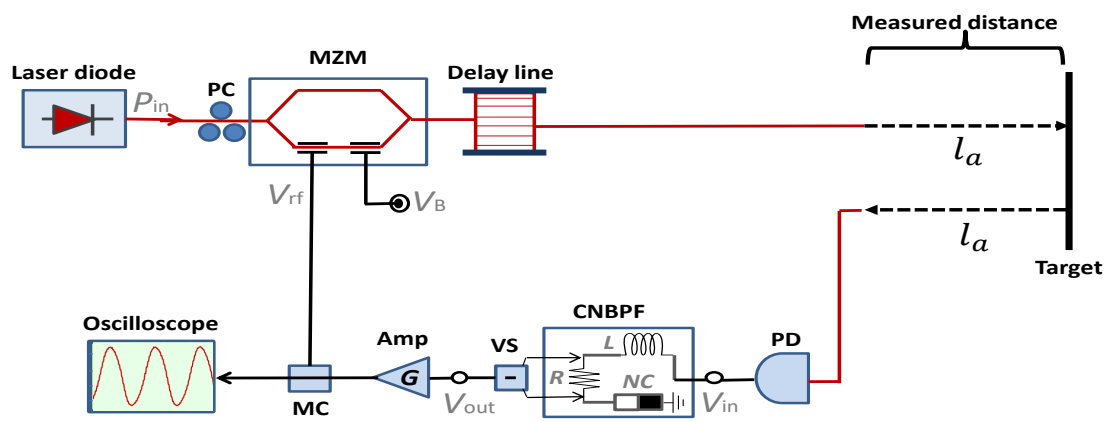

Fig. 8 Setup of the distance measurement using CNOEO. $l_{a}$ is the distance to determine.

\section{Distance measurement using a CNOEO}

5.1 Circuit and principle

When using an OEO for distance measurement, the distance to evaluate $l_{a}$ is the length within the OEO and a target, as shown in Fig. $8[5,20]$. The principle is as follows: the light from the optical delay line travels through the 
free space to the target. The target reflects the light to regain the optical path of the CNOEO. This induces an additional time-delay

$$
\tau_{A}=\frac{2 n_{a} l_{a}}{c}
$$

with $n_{a}$ and $l_{a}$ being the refractive index of air and the distance to measure, respectively. As a result, the oscillation frequency changes and is read by the oscilloscope. The total time-delay $\tau$ of the system of Fig. 8 is the sum of the reference time-delay $\left(\tau_{R}\right)$ and the additional time-delay $\left(\tau_{A}\right)$ given by [20]:

$$
\tau=\tau_{R}+\tau_{A}
$$

where

$$
\tau_{R}=\frac{1}{f_{R}}
$$

with $f_{R}$ representing the reference frequency. Substituting Eq. (16) and (18) in Eq. (17) gives [20]:

$$
\tau=\frac{2 n_{a} l_{a}}{c}+\frac{1}{f_{R}}
$$

From Eq. (19) and applying $\tau=\frac{1}{f_{o s c}}, l_{a}$ is given as follows:

$$
l_{a}=\frac{c}{2 n_{a}}\left(\frac{1}{f_{\text {osc }}}-\frac{1}{f_{R}}\right)
$$

where $f_{\text {osc }}$ is the fundamental frequency of the CNOEO which is determined by the total group delay of the loop $\tau$ read from the oscilloscope (see Eq. (3)). Any N-th frequency mode is harmonic of that fundamental frequency and given by $f_{N}=N f_{o s c}$. Thus $l_{a}$ yields:

$$
l_{a}=\frac{c}{2 n_{a}}\left(\frac{N}{f_{s d}}\left(1+\frac{3 \rho y_{s t}^{2}}{\alpha}\right)^{-\frac{1}{2}}-\frac{1}{f_{R}}\right)
$$

Differentiating Eq. (21), the relative precision of the distance measured is computed as:

$$
\left|\frac{\Delta l_{a}}{l_{a}}\right|=\left(\frac{2 n_{a} l_{a}+c \tau_{R}}{n_{a} l_{a}}\right)\left|\frac{\Delta f_{N}}{f_{N}}\right|
$$

Equation (22) is similar to the one obtained by Wang et al. [20] but with different expression of $f_{N}=N f_{o s c}$ (see Eq. (3)). 


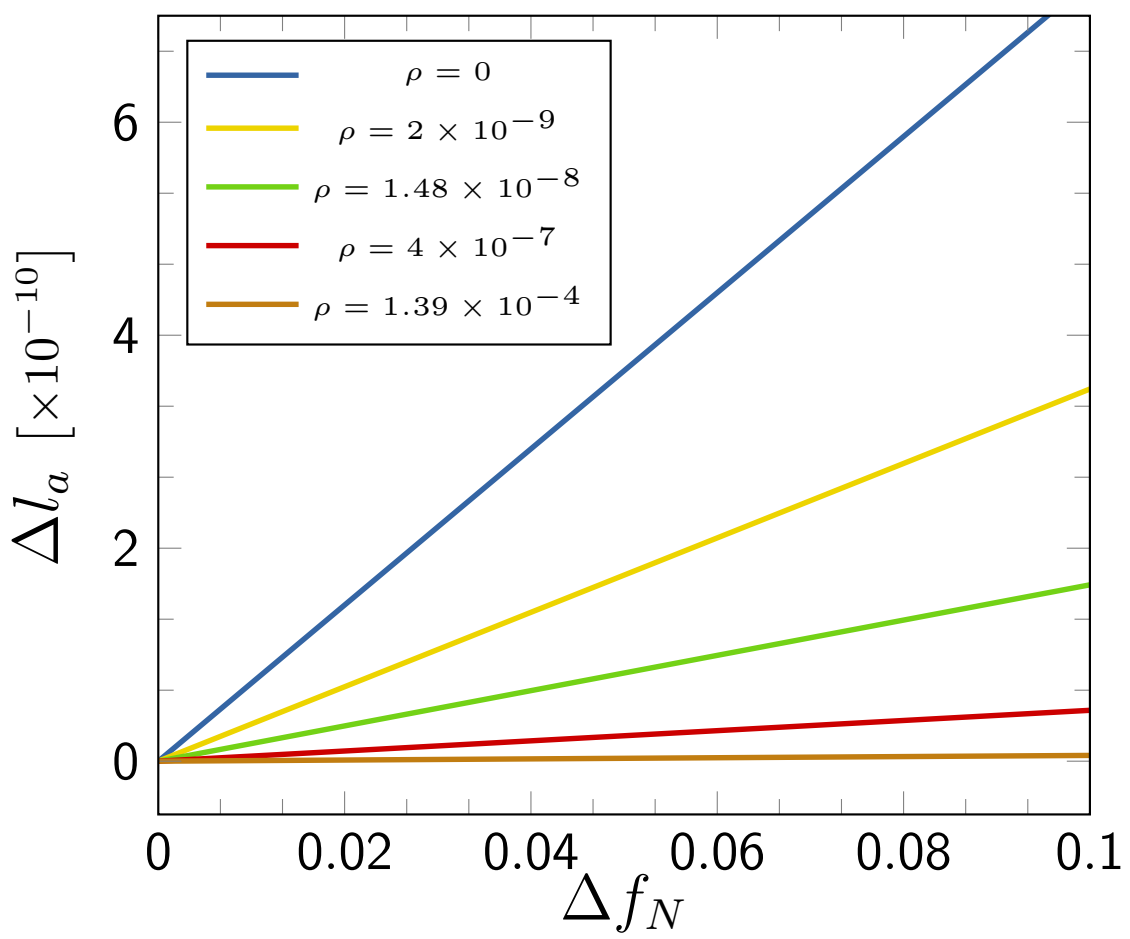

Fig. 9 Variation of the precision of the distance measured $\Delta l_{a}$ versus the variation of the frequency $\Delta f_{N}$. From up to down, $\rho=0, \rho=2 \times 10^{-9}, \rho=1.48 \times 10^{-8}, \rho=4 \times 10^{-7}$, and $\rho=1.39 \times 10^{-4}$.

\subsection{Results}

Figure 9 displays the precision of the distance measured in terms of the variation of the frequency. It can be observed that highly nonlinear CNOEO contributes to obtaining the distance measured with higher precision. That is the standard OEO displays a distance measurement with a large error from the nominal value as $\Delta l_{a}$ is great. However, a CNOEO featuring an important value of the cubic-nonlinear parameter $\rho$ will rather display a distance measured which value is closer to the nominal one as the precision $\Delta l_{a}$ is smaller (see Fig. 9). For instance, for a variation in frequency $\Delta f_{N}=0.04$, the standard OEO $(\rho=0)$ gives a precision of $\Delta l_{a}=3 \times 10^{-10}$ while using a CNOEO with cubic-nonlinear parameter $\rho=1.39 \times 10^{-4}$, the precision drops considerably to $\Delta l_{a}=2.12 \times 10^{-13}$. That is a spanning of about three orders of magnitude. 


\section{Conclusion}

In this work, we have carried out a comparative study of temperature sensing, refractive index sensing, and distance measurement using both the standard and the cubic-nonlinear optoelectronic oscillators. For each of these sensings and the measurement, we have proposed an appropriate setup as well as the analytical expression of the sensing/measurement quantity. It globally comes out that the cubic-nonlinear optoelectronic oscillator (CNOEO) is more sensitive; that is higher precisions in the sensings and the measurement are achieved with the CNOEO than using the standard optoelectronic oscillator. Such precisions become sharper as the values of the cubic-nonlinear parameter become larger. With the CNOEO, results that span several orders of magnitude have been recorded compared to those with the standard OEO. Further investigations will focus on using other novel architectures of OEO [23-26] for other metrological quantities such as strain, load, angular velocity, and more.

\section{References}

1. Larger, L.,: Complexity in electro-optic delay dynamics: Modelling, design and applications. Philos. Trans. Roy. Soc. A, Math. Phys. Eng. Sci., 371, 2799-2802 (2013).

2. Chembo, Y. K., Brunner, D., Jacquot, M., and Larger, L.: Optoelectronic oscillators with time-delayed feedback. Rev. Mod. Phys., 91, 035006-1,51 (2019).

3. Hao, T., Liu, Y., Tang, J., Cen, Q., Li, W., Zhu, N., Dai, Y., Capmany, J., Yao, J., Li, M.: Recent advances in optoelectronic oscillators. Adv. Phot. 2, 044001 (2020).

4. Talla Mbé, J.H., Atchoffo, N.W., Tchitnga, R., Woafo, P.: Dynamics of Time-Delayed Optoelectronic Oscillators With Nonlinear Amplifiers and Its Potential Application to Random Numbers Generation. IEEE J. of Quantum Electron., 57, 5000507, (2021)

5. Zou, X., Liu, X., Li, W., Li, P., Pan, W., Yan, L., Shao, L.: Optoelectronic oscillators (OEOs) to sensing, measurement, and detection. IEEE J. Quantum Electron. 52, 0601116 (2016).

6. Yao, J.,: Optoelectronic oscillators for high speed and high resolution optical sensing. J. Lightw Technol. 35, 3489 - 3497 (2017).

7. Wu, B., Wang, M., Dong, Y., Tang, Y., Mu, H., Li, H., Yin, B., Yan, F., Han, Z.: Magnetic field sensor based on a dual-frequency optoelectronic oscillator using cascaded magnetostrictive alloy-fiber Bragg grating-Fabry Perot and fiber Bragg grating-Fabry Perot filters. Opt. Express., 26, 27628-27638 (2018).

8. Zhang, J., Wang, M., Tang, Y., Ding, Q., Wu, B., Yang, Y., Mu, H., Yin, B., Jian, S.: High-sensitivity measurement of angular velocity based on an optoelectronic oscillator with an intra-loop Sagnac interferometer. Opt. Lett. 43, 2799-2802 (2018).

9. Zhang, J., Wang, M., Tang, Y., Ding, Q., Wang, C., Huang, X., Chen, D., Yann, F.: Angular velocity measurement with improved scale factor based on a wideband-tunable optoelectronic oscillator, IEEE Trans. Instrum. Meas., 70, 7003409 (2021).

10. Liu, L., Gao, H., Ning, T., Pei, L., Zheng, J., Li, J., Xu, J.: High accuracy temperature sensing system exploiting a Sagnac interferometer and an optoelectronic oscillator. Opt. Laser Technol. 123, 1-5 (2019).

11. Zhu, Z., Merklein M., Choi, D.Y., Vu, K., Ma, P., Madden, S.J., and Eggleton, B.J.: Highly sensitive, broadband microwave frequency identification using a chip-based Brillouin optoelectronic oscillator. Opt. Express 27, 12855-12868 (2019).

12. Xie, T., Wang, J., Wang, Z., Ma, C., Yu, Y., Zhu, J., and Yu, J.: Long-range, highprecision, and high-speed absolute distance measurement based on alternately oscillating optoelectronic oscillators. Opt. Express 27, 21635-21645 (2019).

13. Xu, Z., Shu, X., Fu, H.: Fiber Bragg grating sensor interrogation system based on an optoelectronic oscillator loop. Opt. Express 27, 23274-23281 (2019). 
14. Ming, D., Liu, X., Feng, D., Tang, Y., Zhu, T.: Trace copper detection using in-line optical fiber MachZehnder interferometer combined with an optoelectronic oscillator. Opt. Express 29, 23430-23438 (2021).

15. Zhang, N., Wang, M., Wu, B., Han, M., Yin, B., Cao, J., Wang, C.: Temperatureinsensitive magnetic field sensor based on an optoelectronic oscillator merging a MachZehnder interferometer. IEEE Sensors Journal 20, 7053 - 7059 (2020).

16. Feng, D., Kai, L., Zhu, T., Gao, Y., Gao, L., Zhang, J.: High-precision strain-insensitive temperature sensor based on an optoelectronic oscillator." Opt. Express 27, 37532-37540 (2019).

17. Talla Mbé, J.H., Kamaha, J.S.D., Chembo, Y.K., Woafo, P.: Dynamics of wideband time-delayed optoelectronic oscillators with nonlinear filters. IEEE J. Quantum Electron 55, 5000106 (2019).

18. Kamaha, J.S.D., Talla Mbé, J.H., Woafo P.: Routes to chaos and characterization of limit-cycle oscillations in wideband time-delayed optoelectronic oscillators with nonlinear filters. J. Opt. Soc. Am. B. 37, A75-A82 (2020).

19. Zhu, Y., Jin, X., Chi, H., Zheng, S., Zhang, X.: High-sensitivity temperature sensor based on an optoelectronic oscillator. Appl. Opt. 53, 5084-5087 (2014).

20. Wang, J., Yu, J., Miao, W., Sun, B., Jia, S., Wang, W., and Wu, Q.: Long-range, highprecision absolute distance measurement based on two optoelectronic oscillators. Opt. Lett. 39, 4412-4415 (2014).

21. Pham, T.T., Ledoux-Rak, I., Journet, B., Vu, V.Y.: A study on measuring refractive index by using an optoelectronic oscillator." 2014 IEEE Fifth International Conference on Communications and Electronics (ICCE) 10.1109/CCE.2014.6916673, 17-22 (2014).

22. Nguyen, L.D., Nakatani, K., Journet, B.: Refractive index measurement by using an optoelectronic oscillator." IEEE Phot. Tech. Lett. 22, 857 - 859, (2010).

23. Chengui, G.R.G., Talla Mbé, J.H., Talla, A.F., Woafo, P., Chembo, Y.K.: Dynamics of optoelectronic oscillators with electronic and laser nonlinearities. IEEE J. Quantum Electron 54, 5000207 (2018).

24. Chengui, G.R.G, Woafo, P., Chembo, Y.K.: The simplest laser-based optoelectronic oscillator: An experimental and theoretical study. IEEE J. Lightw. Technol. 34, 873-878 (2016).

25. Kouayep, R.M., Talla, A.F., Talla Mbé J.H., Woafo, P.: Bursting oscillations in Colpitts oscillator and application in optoelectronics for the generation of complex optical signals. Opt. Quantum Electron. 52, 291 (2020).

26. Nguewou-Hyousse, H., Chembo, Y.K.: Nonlinear dynamics of miniature optoelectronic oscillators based on whispering-gallery mode electrooptical modulators. Optics Express, 28(21)30656-30674, (2020) 